\title{
Simulation of Microstructure Formation during Thin Film Deposition
}

\author{
Max O. Bloomfield and Timothy S. Cale \\ Focus Center-New York: Rensselaer \\ Rensselaer Polytechnic Institute \\ Troy, NY, USA 12180-3590 \\ bloomm2@rpi.edu,calet@rpi.edu
}

\begin{abstract}
We describe an approach to studying grain structure formation in polycrystalline thin films during deposition from the vapor phase using a "grain continuum" representation of the solid. We employ three codes in concert to track the development of grains while accounting for ballistic transport and surface reaction kinetics. We show that higher precursor reactivities might well lead to higher void fractions near the substrate surface.
\end{abstract}

\section{Introduction}

Grain structure is becoming increasingly important in the performance and reliability of on-chip IC interconnects [1,2]. For example, as critical dimensions of lines approach the electron mean free path in copper, the scattering of carriers by grain boundaries becomes a sizeable term in the RC delay of interconnects [3]. In order to understand and possibly engineer the grain structure of lines, it is important to understand how grain structure develops during deposition. Microstructure is determined by small scale phenomena such as the motion of individual atoms across grain boundaries, adsorption and reaction of precursor molecules on grains, and the diffusion of adatoms on surfaces. However, because these phenomena cannot usually be observed experimentally in evolving systems, researchers have turned to computer simulation to increase understanding of the effects of these underlying mechanisms using the limited available experimental observables.

Consider the problem of vapor deposition of a polycrystalline film. Although there are several methods used for vapor deposition (e.g., PVD, CVD, PECVD, and ALD) most of them are at reduced pressure, giving rise to a mean free path $(\lambda)$ of tens of microns or more. Because characteristic feature dimensions are significantly less than $\lambda$, transport of material is determined by geometric line-of-sight in what is termed Knudsen or ballistic transport. The ballistic transport and reaction model (BTRM) was developed to determine the local fluxes and hence deposition rates in features on patterned surfaces, but it is also applicable to vapor deposition onto grain structures. In this work, we apply a BTRM solver (EVOLVE) [4] to simulate CVD, and observe the response of the system to changes in precursor reactivity. To make these simulations possible, we employ a structural evolution code (PLENTE) [5] and a 3D raytracing code (FVIEW) [6] in concert with EVOLVE.

These types of simulations are potentially valuable in understanding the role of precursor chemistry in determining structure during vapor deposition, and perhaps as 
a tool for engineering structure by manipulating independent variables such as reactant choice and process conditions.

\section{Numerical methods}

The BTRM is represented by a set of integro-differential equations for adsorption, reaction, re-emission from the surface, and line of sight transport of species [4]. For fixed temperature and other reactor setpoints, reactions at the solid-gas interface are determined by the local fluxes of species, the distribution of species adsorbed onto the solid, and the properties of the solid itself. Further, species that strike the solid and do not react may either scatter elastically or be re-emitted with a distribution characteristic of the combination of gas and solid material.

In our simulations, the evolving microstructure is represented implicitly in the form of level set functions by PLENTE [5] before being extracted and represented explicitly as a set of triangular mesh faces. Solution of the BTRM equations on this discretized structure requires knowing the probabilities of line-of-sight transmission ("view factors") between different faces and knowing any externally imposed flux (such as from a source volume overhead) on the system. We use a ray-tracing based code called FVIEW [6] to determine these view factors between faces that are needed in the integrals of the BTRM. EVOLVE uses cyclic substitution to determine reaction rates consistent with these quantities.

Once the reaction rates are known, it is a simple matter of bookkeeping to determine the local speeds of the gas-solid interfaces. These speeds are then "extended" into a higher dimensional domain by PLENTE and used to update the level set representation of the structure [7].

Both the level set and view factor calculations take place in parallel, being distributed over the nodes of a Xeon-based Beowulf cluster, but the BTRM solution is fast enough to be restricted to a single processor. The view factor calculation, although exhibiting linear speed up across 64 processors, still dominates the computation time. For example, even a fairly simple BTRM simulation on 3D surface might involve determining $10^{5}$ view factors.

\subsection{Deposition Results}

Our model system consists of vapor deposition onto a substrate that is unreactive toward a gas phase precursor, except at a limited set of sites which act as nucleation "traps". These reactive sites might be defects on the surface or sites functionalized during a pre-deposition treatment, such as the use of water vapor in the early stages of Al CVD [8]. We examine the effect that the reactivity of the precursor on the depositing film has on the resulting microstructure. It is difficult to experimentally isolate and study the effects of reactivity on microstructure because it is difficult to manipulate it as an independent variable. Thus to evaluate this model, we relate our results to analogs in simulation studies of roughening of surfaces during deposition, such as those in Ref. 9, and to the large body of experimental data and simulation of conformality of continuous thin films in topographic features. (See Ref. 4 and references therein.)

Species fluxes to each point on the surface come both directly from the source and from re-emission of unreacted precursor from the surface. The mechanistic details of the deposition chemistry are not important for the purposes of this paper; however, 
suppose that the overall reaction results in the irreversible deposition of copper and is considered to have a rate $r$ of the form:

$$
r=k_{0} \exp \left(-\frac{E_{A}}{k T}\right) f(\boldsymbol{\eta})
$$

Here, $k_{0}$ is the pre-exponential factor, $E_{A}$ is the activation energy, $k$ the Boltzmann constant, $T$ the temperature, and $f$ a function of the fluxes $\boldsymbol{\eta}$ that is characteristic of the underlying mechanism. Suppose the rate-limiting step remains the same and the mechanism does not change $(f(\boldsymbol{\eta})$ stays about the same) as the reactivity of the precursor is adjusted by changing the temperature. Though deposition reactions are in general complex, including several "mechanistic" steps, it suffices for this paper to consider simple first order reactions. In this case, $f(\eta)=\eta$ and the probability of reaction is given by $k_{0} \exp \left(-E_{A} / k T\right)$. This quantity is commonly called the sticking factor and may be interpreted as the probability that an incoming precursor molecule will react or "stick" to the surface. Figure 1 shows three outcomes of deposition, on a fixed nucleation pattern, with sticking factors on $\mathrm{Cu}$ of $1.0,0.5$ and $10^{-3}$ (and zero on the substrate). From analyses of these results (such as by the $2 \mathrm{D}$ cross-sections pictured below each $3 \mathrm{D}$ picture) it is seen that the slightly and moderately reactive precursors create a denser grain structure with less void volume due to shadowing and less prominent surface seams than the highly reactive precursor. It has been observed in these and other simulations that nuclei that are positioned in the lee of larger grains become completely shadowed by their larger neighbors and are "choked off" $[10,11]$.

The observed trend of voiding behavior, i.e., increasing void volume with increasing reactivity, is the $3 \mathrm{D}$ analog of the behavior studied in $2 \mathrm{D}$ by Singh and Shaqfeh [9] for continuous surfaces. There it was seen that higher sticking factors allowed valleys to pinch off before filling completely, leaving characteristic voids. This trend is also observed in the large body of experimental and simulation work for conformality in features [ 4 and references therein] Although the structures in the current work are granular, the geometric issues are the same as in the continuous film studies, with shadowing being responsible for voids developing in concave structures.

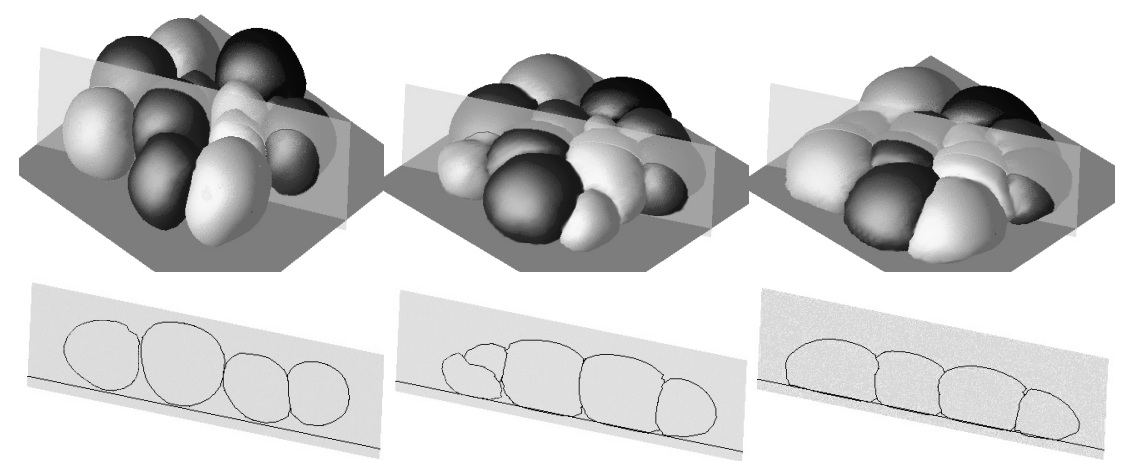

Figure 1. 3D simulated grain structure and cross sections after short depositions of material with sticking factors (on $\mathrm{Cu}$ ) of (left) 1 , (center) 0,5 , and (right) $10^{-3}$ as noted in the text. The film exhibits increasing density (decreasing void volume) as deposition becomes more isotropic, from the left with a prominent void structure to the right with almost no visible voids. 


\section{Conclusions}

We used the ballistic transport and reaction model with material-dependent reaction rates to model a simple deposition process. These simulations indicate that the details of grain structures can be affected by precursor reactivity on the depositing surface, as well as by the location and size distribution of deposited nuclei. The computational method used extended the applicability of a trio of already existing codes, working in concert to simulate an important physical system.

\section{Acknowledgments}

Support from MARCO, DARPA, and NYSTAR through the Interconnect Focus Center is gratefully acknowledged.

\section{References}

1. W. Steinhoegl, G. Schindler, G. Steinlesberger, M. Traving, M. Engelhardt, 2003 IEEE International Conference on Simulation of Semiconductor Processes and Devices, 2003, pp. 27-30.

2. J. Proost, J. D'Haen, M. Jin, B. Verlinden, Scripta Materialia 50(2), 267-71 (2004).

3. Intl. Tech. Roadmap for Semiconductors http://public.itrs.net/

4. T.S. Cale, T.P. Merchant, L.J. Borucki, A.H. Labun, Thin Solid Films 365(2), 152-75 (2000).

5. M.O. Bloomfield, D.F. Richards, T.S. Cale, Philisophical Mag. 83(31-34), 3549-68 (2003).

6. T.S. Cale, T.P. Merchant, L.J. Borucki, in Semiconductor Process and Device Performance Modelling, Mat. Res. Soc., v 490, 1998, p. 201-212.

7. S. Osher, J. Sethian, J. Comp. Phys. 79, 12-49 (1988).

8. D. Yang, J. Hong, D. F. Richards, and T. S. Cale, J. Vac. Sci. Technol. B 20(2), 495-506 (2002).

9. V.K. Singh and E.S.G. Shaqfeh, J. Vac. Sci. Technol A 11(3) 557-568 (1993).

10. F. Ying, R.W. Smith, D.J. Srolovitz, Appl. Phys. Let., 69 (20), 3007-9 (1996).

11. T.J. Smy, S.K. Dew, R.V. Joshi, J. Vac Sci. Technol. A, 19 (1), 251-61 (2001). 